\title{
sciendo
}

Journal of Research in Physics

Vol. 39, No. 1, $1-22(2018)$

doi:10.2478/jrp-2018-0001

\section{Theory and experiments of liquid phase epitaxial growth Part 1}

\author{
Nikolay S. Peev \\ Acad. G. Nadjakov Institute of Solid State Physics, Bulgarian Academy of Sceinces \\ E-mail: nasta_first@abv.bg
}

Received: June 1, 2016

\begin{abstract}
Characterization of the liquid phase epitaxial growth is in the scope of the present paper. The determination of the main parameters of the process such as the stationary growth rate, the time constant, the stationary supercooling, the kinetic coefficient, the initial growth rate, the crystallization mechanism are under consideration. Pursuing this aim a differential equation has been obtained which allows obtaining the dependences of the supersaturation, the growth rate and the growth layer thickness on the duration of growth. These dependences allow the determination of the above mentioned process parameters. Two mechanisms of crystallization are considered - the normal growth and the growth assisted by the screw dislocations. The two dimensional nuclei growth has not been considered because an analytical solution of the equation was not possible. Some of the theoretical contentions are confirmed by the experiments. Using the obtained values of the parameters dependences are defined. Good agreement between theory and experimental results is observed.
\end{abstract}

Key words: liquid phase epitaxial growth, crystallization mechanisms, theory and experiments.

\section{Introduction}

The liquid phase epitaxy (LPE) is the basic technology for the thin layer growth of garnets [1-3], semiconductors ( $\mathrm{GaAs}, \mathrm{Si}, \mathrm{GaN}$, ternary and quaternary compounds and others) [4-17], superconductors (YBCO) [18-27] and materials for wave guide applications $\left(\mathrm{LiYF}_{4}, \mathrm{LNbO}_{3}\right)[28,29]$. In different directions there are significant achievements in the field of LPE layers growth - the grown layers having thickness in the range of $2 \mathrm{~nm}[15,16]$; large area layers being deposited (up to 3 inches) [24]; the growth of high purity layers $[12,17]$. Using LPE techniques single crystals of $\mathrm{GaN}, \mathrm{SiC}$ and YBCO with thickness up to $3 \mathrm{~mm}$, with impurities concentration of $4 \cdot 10^{14} \mathrm{~cm}^{-3}$ and dislocations density of $2 \cdot 10^{5} \mathrm{~cm}^{-3}$ are grown $[4-8,21,26,27]$. 
Layers of high quality can be grown using LPE technology, comparable to that deposited by the vapor phase epitaxial methods. A better understanding of the liquid phase epitaxial process will lead to better practical results, obtained by them.

Despite the significance of obtaining practical results, the knowledge of the LPE process remains insufficient until now. There are questions that are still not answered by the theory $[30,31]$. Theoretically only the case of dilute components deposition is considered - what is the behavior of the components having a high amount in the liquid phase?Many parameters of the process have not been established, such as the kinetic coefficient $\mu$, time constant $\tau$, crystallization mechanism and others.

The present paper is in fact a continuation of references [32,33]. Two kinds of components have been distinguished: the $i$-components and the $j$-components [32-38]. The $i$-components are the dilute components of the process - their concentration in the liquid phase is smaller than that in the solid phase: $C_{i}^{L}<C_{i}^{S}$. The consequence of this fact is that their concentration decreases with time permanently, $d C_{i}^{L} / d t<0$, and the liquid phase becomes increasingly deficient in these components [32-38]. The $j$-components have a high content in the liquid phase: their concentration in the liquid phase is higher than that in the solid one: $C_{j}^{L}>C_{j}^{S}$ and therefore it increases with time permanently $d C_{j}^{L} / d t>0$, the liquid phase becomes more and more abundant of $j$-components. The mass transport of $i$-components occurs always toward the crystal surface but for the $j$-components it is in the opposite direction. That is the reason why the liquid phase is supersaturated with respect to the $i$-components and superheated with respect to the $j$-components [32-38].

The LPE growth is a strongly correlated process - the deposition of the components onto the crystal surface occurs in proportions, subjected to the stoichiometry of the grown compound [32-37]. This requirement is always fulfilled and does not depend on the crystallization mechanism, on the intensity of the diffusion mass transport or the process growth regime, i.e. whether diffusion limited or kinetically limited growth has taken place. The kinetic growth regime takes place at the beginning of the process, when the growth is dominated by the surface kinetics. During this regime the transition of the system from the initial state (presented by the initial supersaturation $\Delta C_{i 0}^{L}$ ) to the stationary growth (presented by the stationary value of the supersaturation $\Delta C_{i}^{L^{*}}$ ) occurs. During this period the deviation from the stationary conditions is very strong and the growth rate $v_{g r}$ is much larger than the stationary growth rate $v_{g r}^{*}$. The stationary growth conditions are observed at greater durations of the process $[32,33]$ and the value of the growth rate $v_{g r}^{*}$ is determined by the intensity of the diffusion mass transport - the growth is dominated by the diffusion.

In the scope of the present paper the basic parameters of the liquid phase epitaxial growth are presented: the time constant of the process $\tau$ (which characterizes the rate of transition from the kinetic growth regime to the stationary one), the kinetic coefficient $\mu$, the stationary growth rate $v_{g r}^{*}$, stationary supercooling $\Delta T^{*}$, the initial growth rate $v_{g r .0}$. The normal and the dislocation mechanisms of crystallization are considered. The twodimensional nuclei growth has not been considered, because the corresponding equation can't be solved analytically. The three most widely used LPE techniques are taken into account: supercooling (Su.), equilibrium cooling (Eq.) and step cooling (St.). Exotic techniques - capillary-LPE and electro-LPE, have not been discussed.

In the presented approach each component is presented by its equilibrium concentration $C_{i . e q}^{L}$, the own true concentration $C_{i}^{L}$, the own supersaturation $\Delta C_{i}^{L}$, the own density in the solid phase $\gamma_{i}^{S}$ and the own growth rate $v_{\text {gr.i. }}$. The growth rate of different components 
is the same and is equal to the growth rate of the compound itself: $v_{g r .1}=v_{g r .2}=v_{g r .3}=$ $\ldots=v_{g r . n_{0}}=v_{g r}^{\text {comp. }}[33-35,38]$. The presented approach allows the investigation of the dependences of supersaturation $\Delta C_{i}^{L}$, growth rate $v_{g r}$ and layer thickness $d$ versus time $t$, versus initial supersaturation $\Delta C_{i .0}^{L}$ and versus cooling rate $v_{T}$. The time dependence of the main parameters of the process (supersaturation, growth rate and layer thickness) is considered in the present part of this paper. The dependence of the supersaturation versus time $\Delta C_{i}^{L}(t)$ has been considered in [32] - it is in fact the value of the supersaturation averaged over the whole volume of the melt. The time dependence of the growth rate $v_{g r}(t)$ and layer thickness $d(t)$ are established using the already known dependence $\Delta C_{i}^{L}(t)$ [32] and they are the subject of the present paper. In the second part of the paper the dependences of the supercooling $\Delta C_{i}^{L}$, growth rate $v_{g r}$ and growth layer thickness $d$ on the initial supercooling $\Delta C_{i 0}^{L}$ and on the cooling rate $v_{T}$ will be considered (theoretical and experimental). Experimental investigations [28] of the growth layer thickness on the initial supercooling $d\left(\Delta C_{i 0}^{L}\right)$ at step cooling technique is going to be discussed in that part.

The diffusion mass transport has not been taken into account. First of all the diffusion dominates the growth process during the stationary regime - at the kinetics limited regime the surface processes are responsible for the growth rate. Because of that the application of the diffusion equation to the growth process [30,31] does not allow the determiniation of the crystallization mechanism. The diffusion mass transport and its effect on the growth process will be the subject of future investigation which must take into account the already established requirements, namely: 1) the mass transport of each component must subject to the stoichiometry of the growth compound $[32,33,36]$ and 2) some of the components (the dilute components - the $i$-components) diffuse toward the interface boundary but the others (the $j$-components) - in the opposite direction [32-36]. The present investigations are based on the circumstance that the growth process is strongly correlated in the sense that the deposition of the components occurs always in proportions subjected to the stoichiometry of the compound itself, which is fulfilled at any mechanisms of growth, at any growth regime (kinetically growth regime or stationary one).

\section{Theory}

A liquid phase with $n_{0}$ - components is in contact with a $n_{0}$ - components solid phase through an interface boundary - the components in both phases are the same. The composition of the liquid phase with very small deviations is determined by the liquidus surface - the deviations are caused by the supercooling, which is as a rule very small. The composition of the solid phase is determined by the stochiometric composition of the compound and does not change during growth. During growth, caused by the temperature decrease, the parameters of components undergo significant changes - the masses of all components decrease by $d m_{i}$ within the time $d t, i \in\left(1, n_{0}\right)$. For the dilute components the real concentration $C_{i}^{L}$ and the equilibrium one $C_{i . e q}^{L}$ within the same time $d t$ decrease too, but for the high concentrated components both concentrations increase. For the dilute components the liquid phase is super cooled but for those components, having high concentration in the liquid phase, it is superheated [32-37].

The supersaturation $\Delta C_{i}^{L}$ of the dilute components and the growth rate $v_{g r}$ follow the changes of both concentrations - the true and the equilibrium ones: $\Delta C_{i}^{L}=C_{i}^{L}-$ $C_{i . e q}^{L}, d\left(\Delta C_{i}^{L}\right)=d C_{i}^{L}-d C_{i . e q}^{L}, v_{g r} \sim \Delta C_{i}^{L}$, where $C_{i}^{L}$ and $C_{i . e q}^{L}$ are the true and the equilibrium concentrations of the $i$-component, respectively. The time dependence of the 
real concentration $C_{i}^{L}$ depends on the liquidus surface, the growth temperature $T_{0}$, the growth rate $v_{g r}$, the mass of the liquid phase and the substrate's area [37, 38]:

$$
d C_{i}^{L}=\frac{C_{k}^{L}}{C_{k}^{S}}\left(C_{i}^{S}-C_{i}^{L}\right) \frac{d m_{k}^{L}}{m_{k}^{L}}=\frac{\gamma_{0}^{S} S_{0} M_{k}}{M_{0} m_{k}^{L}} C_{k}^{L}\left(C_{i}^{S}-C_{i}^{L}\right) v_{g r} d t
$$

The equilibrium concentration subjected to the temperature decrease occurring with a cooling rate $v_{T}$ changes with time as $[37,38]$ :

$$
d C_{i . e q}^{L}=\frac{d T}{k_{i}}=\frac{v_{T} d t}{k_{i}}
$$

It has been supposed that within the volume of the liquid phase no temperature gradient occur and the equilibrium concentration is the same over the whole volume. Equations are valid in a coordinate system with $x$-axis normal to the crystal surface and an onset, disposed on the crystal surface. The concentration gradients follow the direction of $x$-axis - in other directions there are no gradients.

Equation 1 is valid within the frames of a growth process. It has been obtained using the dependence of the change of concentration $d C_{i(\text { or } j)}^{L}$ of an arbitrary component ( $i$ or $j$ ) on the change of its mass $d m_{i(o r j)}^{L}$. Both changes, $d C_{i(\text { or } j)}^{L}$ and $d m_{i(o r j)}^{L}$ occur within the time $d t$ and are the consequence of growth [34,35,38]. The change of the mass $d m_{i(o r j)}^{L}$ of the liquid phase with time during growth is always negative and provides the growth rate $v_{g r}$. The change of the concentration $d C_{i}^{L}$ of $i$-components with time is negative but the change of $j$-components concentration $d C_{j}^{L}$ is positive, as mentioned above. The correlation $d C_{i(\text { or } j)}^{L} \Leftrightarrow d m_{i(\text { or } j)}^{L}$ is valid at any mechanism of growth, at any growth regime (kinetically or stationary) and any growth technique (supercooling, step cooling, equilibrium cooling).

Additionally, the strong correlation of the process has been taken into account, which means that the amount of the incorporation of the components into the solid phase subjects to the stoichiometry of the growth compound [35-38]. As a consequence of growth the changes of the masses $d m_{m}^{L} \Leftrightarrow d m_{l}^{L}$ (at any values of the subscripts $m$ and $l$ ) within the time $d t$ occur in strict proportions, subjected to the stoichiometry. The correlation of the masses is valid also at any mechanism of growth, at any growth regime and any growth technique. The composition of the grown solid phase does not change during growth the compound has a constant composition. Due to the strong correlation the behavior of each component depends on the behavior of all other components, which allows the introduction of a basic component, denoted by subscript " $\mathrm{k}$ " - each component may be a basic one. Investigating the behavior of the basic component one may study the behavior of the others [32, 35].

From the above mentioned follows that equation 1 is valid at any mechanism of growth, any growth regime and any growth technique. This equation concerns the behavior of an arbitrary $i$-component. The $i$-components have been preferred because they have a real mass transport toward the crystal surface which has, as a consequence, a real supersaturation over the crystal. The $j$-components diffuse away from the crystal surface, deep into the liquid phase volume, and for these components the liquid phase is always superheated [32-38]. Equation 1, therefore, provides information how the actual concentration of an arbitrary $i$-component (dilute component) depends on the time variations of the temperature, the growth rate, the liquid phase mass, the growth area, the liquidus surface features. 
The differential equation concerning supersaturation is as follows[32, 35, 37, 38]:

$$
d\left(\Delta C_{i}^{L}\right)=d C_{i}^{L}-d C_{i . e q}^{L}=\left[\frac{v_{T}}{k_{i}}-\frac{\gamma_{0}^{S} S_{0} M_{k}}{M_{0} m_{k}^{L}} C_{k}^{L}\left(C_{i}^{S}-C_{i}^{L}\right) v_{g r}\right] d t
$$

The growth rate depends on the supersaturation also. Equation 3 is valid for each dilute component. It has been solved in cases of normal mechanism of growth and for screw dislocations growth mechanism. In both cases the growth rate depends on the supersaturation by different manner through the following relations:

$$
\begin{aligned}
& v_{g r}=\mu_{i . N} \Delta T=\mu_{i . N} k_{i} \Delta C_{i . N}^{L} \quad \rightarrow \quad \text { normal mechanism of growth, } \\
& v_{g r}=\mu_{i . D}(\Delta T)^{2}=\mu_{i . D}\left(k_{i} \Delta C_{i . D}^{L}\right)^{2} \quad \rightarrow \quad \text { dislocation mechanism of growth }
\end{aligned}
$$

The solution of equation 3 provides expressions for supersaturations at both growth mechanisms and each growth technique - the supercooling technique $\Delta C_{i}^{L(S u .)}(t)$, the equilibrium cooling $\Delta C_{i}^{L(E q .)}(t)$ and the step cooling $\Delta C_{i}^{L(S t .)}(t)$. Using the corresponding expression for the supersaturation and by the corresponding equation 4 the growth rate has been obtained $-v_{g r}^{(S u .)}(t), v_{g r}^{(E q .)}(t)$ and $v_{g r}^{(S t .)}(t)$. By integrating the growth rate over time one obtains the change of the layer thickness with the duration of growth $d^{(S u .)}(t)$, $d^{(E q .)}(t)$ and $d^{(S t .)}(t): d(t)=\int_{0}^{t} v_{g r}(\tau) d \tau$.

During the stationary regime the growth rate and the supersaturation do not change with time - they are almost constant. Equation 3 using equations 1 and 2 provides the stationary growth rate $v_{g r}^{*}[38]$ :

$$
\begin{aligned}
d\left(\Delta C_{i}^{L}\right) & =d C_{i}^{L}-d C_{i . e q}^{L}=0 \Rightarrow d C_{i}^{L} \equiv d C_{i . e q}^{L} \\
v_{g r}^{*} & =\frac{M_{0} m_{k}^{L^{*}}}{k_{i} \gamma_{0}^{S} S_{0} M_{k} C_{k}^{L^{*}}\left(C_{i}^{S}-C_{i}^{L^{*}}\right)} v_{T}
\end{aligned}
$$

$v_{g r}^{*}, m_{i}^{L^{*}}, C_{i}^{L^{*}}$ and $C_{k}^{L^{*}}$ are the stationary values of the corresponding quantities. It is worth to point out that in the last expression there are no kinetic coefficients, which means that in stationary regime the growth process does not depend on the growth mechanism. It is dominated by the diffusion mass transport.

\subsection{Normal growth mechanism}

Since the dependence of the growth rate on the supersaturation is linear (eq. 4), eq. 3 takes the form [38]:

$$
d\left(\Delta C_{i}^{L}\right)=d C_{i}^{L}-d C_{i . e q}^{L}=\left[\frac{v_{T}}{k_{i}}-\mu_{i . N} k_{i} \frac{\gamma_{0}^{S} S_{0} M_{k}}{M_{0} m_{k}^{L}} C_{k}^{L}\left(C_{i}^{S}-C_{i}^{L}\right) \Delta C_{i . N}^{L}\right] d t
$$

It is a linear differential equation providing the following solution, valid for all the growth techniques [38]:

$$
\begin{aligned}
\Delta C_{i}^{L}(t) & =\Delta C_{i . N}^{L}-\frac{B_{0}}{A_{i . N}} \exp \left(-A_{i . N} t\right) \\
A_{i . N} & =\mu_{i . N} k_{i} \frac{\gamma_{0}^{S} S_{0} g_{k} M_{k}}{M_{0} m_{k}^{L}} \frac{C_{k}^{L}}{C_{k}^{S}}\left(C_{i}^{S}-C_{i}^{L}\right)=\frac{1}{\tau_{i . N}} \\
\Delta C_{i . N}^{L} & =\Delta C_{i . N}^{L^{*}}=\frac{v_{T}}{A_{i . N} k_{i}}=\frac{v_{T} \tau_{i . N}}{k_{i}} \Rightarrow v_{T} \tau_{i . N}=k_{i} \Delta C_{i . N}^{L^{*}}
\end{aligned}
$$


Using the corresponding initial and boundary conditions, the dependences of the supersaturation on time for all three growth techniques have been obtained. The supercooling technique takes place at the following conditions: liquid phase, initially super cooled by $\Delta C_{i .0}^{L}, \Delta C_{i}^{L}(t=0)=\Delta C_{i .0}^{L}$, and temperature decreases with velocity $v_{T}$ (it means $\left.\Delta C_{i . N}^{L} \neq 0\right)$. At the equilibrium cooling the initial supercooling is zero and the growth occurs with the aid of supercooling, provided by the temperature decrease: $v_{T} \neq 0$ and $\Delta C_{i .0}^{L}=0$. At the step cooling technique the process is isothermal, at constant temperature, and the layer grows in consequence of the initial supercooling: $v_{T}=0$ and $\Delta C_{i .0}^{L} \neq 0$. The respective solutions are as follows [38]:

- supercooling:

$$
\Delta C_{i . N}^{L(S u .)}(t)=\Delta C_{i . N}^{L(S u .)}-\left(\Delta C_{i . N}^{L(S u .)}-\Delta C_{i .0}^{L}\right) \exp \left(-\frac{t}{\tau_{i . N}^{(S u .)}}\right)
$$

- equilibrium cooling:

$$
\Delta C_{i . N}^{L(E q .)}(t)=\Delta C_{i . N}^{L(E q .)}-\left[1-\exp \left(-\frac{t}{\tau_{i . N}^{(E q .)}}\right)\right]
$$

- step cooling:

$$
\Delta C_{i . N}^{L(S t .)}(t)=\Delta C_{i . N}^{L(S t .)} \exp \left(-\frac{t}{\tau_{i . N}^{(S t .)}}\right)
$$

where $\tau_{i . N}$ are the corresponding time constants and $\Delta C_{i . N}^{L}$ is the stationary value of the supersaturation: $\Delta C_{i . N}^{L}=\Delta C_{i . N}^{L^{*}}$ [38]. Using equation 4 one obtains the expressions for the corresponding growth rate:

$$
\begin{gathered}
v_{g r N}^{(S u .)}(t)=v_{g r N}^{(S u .)^{*}}-\left(v_{g r N}^{(S u .)^{*}}-v_{g r 0 N}\right) \exp \left(-\frac{t}{\tau_{i . N}^{(S u .)}}\right) \\
v_{g r N}^{(E q .)}(t)=v_{g r N}^{(E q .)^{*}}\left[1-\exp \left(-\frac{t}{\tau_{i . N}^{(E q .)}}\right)\right] \\
v_{g r N}^{(S t .)}(t)=v_{g r .0 N} \exp \left(-\frac{t}{\tau_{i . N}^{(S t .)}}\right)
\end{gathered}
$$

Integrating equations 11-13 one obtains expressions concerning the layer growth thickness versus growth time:

$$
\begin{gathered}
d_{N}^{(S u .)}(t)=\int_{0}^{t} v_{g r N}(\tau) d \tau=v_{g r}^{*} t+\left(v_{g r 0 N}-v_{g r}^{*}\right) \tau_{i . N}^{(S u .)}\left[1-\exp \left(-\frac{t}{\tau_{i . N}^{(S u .)}}\right)\right] \\
d_{N}^{(E q .)}(t)=v_{g r}^{*} t-v_{g r}^{*} \tau_{i . N}^{(E q .)}\left[1-\exp \left(-\frac{t}{\tau_{i . N}^{(E q .)}}\right)\right] \\
d_{N}^{(S t .)}(t)=v_{g r .0 N} \tau_{i . N}\left[1-\exp \left(-\frac{t}{\tau_{i . N}^{S t .}}\right)\right]
\end{gathered}
$$


The following superpositions are observed in all expressions:

$$
\begin{gathered}
\Delta C_{i N}^{L(S u .)}(t)=\Delta C_{i N}^{L(E q .)}(t)+\Delta C_{i N}^{L(S t .)}(t), \\
v_{g r N}^{(S u .)}(t)=v_{g r N}^{(E q .)}(t)+v_{g r N}^{(S t .)}(t), \\
d_{g r N}^{(S u .)}(t)=d_{g r N}^{(E q .)}(t)+d_{g r N}^{(S t .)}(t) .
\end{gathered}
$$

\subsection{Dislocation Growth Mechanism}

In the case of dislocation growth mechanism the growth rate depends on the supersaturation by a parabolic relation (eq. 4) and the corresponding differential equation is as follows [38]:

$$
d\left(\Delta C_{i}^{L}\right)=d C_{i}^{L}-d C_{i . e q}^{L}=\left[\frac{v_{T}}{k_{i}}-\mu_{i . D} k_{i}^{2} \frac{\gamma_{0}^{S} S_{0} M_{k}}{M_{0} m_{k}^{L}} C_{k}^{L}\left(C_{i}^{S}-C_{i}^{L}\right) \Delta C_{i . D}^{L^{2}}\right] d t
$$

The initial and boundary conditions of the different growth techniques are the same with the above mentioned and the corresponding expressions concerning supersaturations are [38]:

- supercooling:

$$
\begin{array}{r}
\Delta C_{i}^{L(S u .)}(t)=\Delta C_{i . D}^{L} \frac{\Delta C_{i 0}^{L} \operatorname{ch}\left(\frac{t}{\tau_{i . D}^{(S u .)}}\right)+\Delta C_{i . D}^{L} \operatorname{sh}\left(\frac{t}{\tau_{i . D}^{(S u .)}}\right)}{\Delta C_{i 0}^{L} \operatorname{sh}\left(\frac{t}{\tau_{i . D}^{(S u .)}}\right)+\Delta C_{i . D}^{L} \operatorname{ch}\left(\frac{t}{\tau_{i . D}^{(S u .)}}\right)} \\
G_{i D}=\mu_{i . D} k_{i}^{2} \frac{\gamma_{0}^{S} S_{0} g_{k} M_{k}}{M_{0} m_{k}^{L}} \frac{C_{k}^{L}}{C_{k}^{S}}\left(C_{i}^{S}-C_{i}^{L}\right)=\frac{1}{\tau_{00 i . D}}\left(s^{-1}\right) \\
\tau_{i . D}^{(S u .)}=\left(\frac{k_{i}}{v_{T} G_{i . D}}\right)^{1 / 2} ; \quad \Delta C_{i . D}^{L}=\Delta C_{i . D}^{L^{*}}=\left(\frac{v_{T}}{k_{i} G_{i . D}}\right)^{1 / 2} \rightarrow \\
\rightarrow \Delta C_{i . D}^{L} \tau_{i . D}=\frac{1}{G_{i . D}}=\tau_{00 i . D}, \quad k_{i} \Delta C_{i . D}^{L^{*}}=v_{T} \tau_{i . D}^{(S u .)}
\end{array}
$$

- equilibrium cooling:

$$
\Delta C_{i}^{L(E q .)}(t)=\Delta C_{i . D}^{L} t h\left(\frac{t}{\tau_{i . D}^{(E q .)}}\right)
$$

- step cooling:

$$
\Delta C_{i}^{L(S t .)}(t)=\frac{\Delta C_{i 0}^{L}}{G_{i . D} \Delta C_{i 0}^{L} t+1}=\frac{\Delta C_{i 0}^{L} \tau_{00 i . D}}{\Delta C_{i 0}^{L} t+\tau_{00 i . D}}
$$

In the same way one obtains the equations concerning the growth rate and the layer thickness:

$$
v_{g r D}^{(S u .)}(t)=\mu_{i . D} k_{i}^{2}\left(\Delta C_{i}^{L}\right)^{2}=v_{g r}^{*}\left(\frac{\Delta C_{i 0}^{L} \operatorname{ch}\left(\frac{t}{\tau_{i . D}^{(S u .)}}\right)+\Delta C_{i . D}^{L} \operatorname{sh}\left(\frac{t}{\tau_{i . D}^{(S u .)}}\right)}{\Delta C_{i 0}^{L} \operatorname{sh}\left(\frac{t}{\tau_{i . D}^{(S u .)}}\right)+\Delta C_{m D}^{L} \operatorname{ch}\left(\frac{t}{\tau_{i . D}^{(S u .)}}\right)}\right)^{2}
$$




$$
\begin{gathered}
v_{g r D}^{(E q .)}(t)=v_{g r}^{*} t h^{2}\left(\frac{t}{\tau_{i . D}^{(E q .)}}\right)=v_{g r}^{*}\left(\frac{e^{2 t / \tau_{i . D}^{(E q .)}}-1}{e^{2 t / \tau_{i . D}^{(E q .)}}+1}\right)^{2} \\
v_{g r D}^{(S t .)}(t)=\mu_{i . D} k_{i}^{2}\left(\frac{\Delta C_{i 0}^{L}}{G_{i . D} \Delta C_{i 0}^{L} t+1}\right)^{2}=\frac{v_{g r 0 D}}{\left(G_{i . D} \Delta C_{i 0}^{L} t+1\right)^{2}} \\
d_{D}^{(S u .)}(t)=v_{g r}^{*} t+\frac{v_{g r}^{*} \tau_{i . D}^{(S u .)}}{x_{D}^{(S u .)}}-v_{g r}^{*} \tau_{i . D}^{(S u .)} \frac{\Delta C_{i 0}^{L} c h\left(\frac{t}{\tau_{i . D}^{(S u .)}}\right)+\Delta C_{i . D}^{L^{*}} s h\left(\frac{t}{\tau_{i . D}^{(S u .)}}\right)}{C_{i 0}^{L} s h\left(\frac{t}{\tau_{i . D}^{(S u .)}}\right)+\Delta C_{i D}^{L^{*}} c h\left(\frac{t}{\tau_{i . D}^{(S u .)}}\right)} \\
d_{D}^{(E q .)}(t)=v_{g r}^{*} t-v_{g r}^{*} \tau_{i . D}^{(E q .)} \frac{e^{2 t / \tau_{i . D}^{(E q .)}}-1}{e^{2 t / \tau_{i . D}^{(E q .)}}+1}=v_{g r}^{*} t-v_{g r}^{*} \tau_{i . D}^{(E q .)} t h\left(\frac{t}{\tau_{i . D}^{(E q .)}}\right) \\
d_{D}^{(S t .)}(t)=\mu_{i . D} k_{i}^{2} \frac{\Delta C_{i 0}^{L^{2}} t}{G_{i . D} \Delta C_{i 0}^{L} t+1}=\frac{v_{g r 0 D} t}{G_{i . D} \Delta C_{i 0}^{L} t+1}
\end{gathered}
$$

Analogous to the normal mechanism, the quantity $\tau_{i . D}^{(E q .)}$ is the time constant of the process, characterizing the transition from the initial state (given by the initial supersaturation $\Delta C_{i 0}^{L}$ ) to the stationary state (given by the stationary supersaturation $\Delta C_{i . D}^{L^{*}}$ ). The time constant differs from the time constant of the normal growth - in the case of dislocation growth the time constant depends on the cooling rate (eq. 18), while in the case of normal growth it does not (eq. 7) [38]. The quantity $\Delta C_{i . D}^{L}$ is in fact the stationary value of the supersaturation $\Delta C_{i . D}^{L^{*}}, \Delta C_{i . D}^{L}=\Delta C_{i . D}^{L^{*}}$ (eq. 18) [38]. In the case of dislocation mechanism of growth no superpositions are observed between the values of the supersaturations, the growth rates and the layer thicknesses.

The relation $k_{i} \Delta C_{i}^{L^{*}}=v_{T} \tau_{i}^{(S u .)}$ is remarkable, since it can be observed in both mechanisms of growth (eq. 7 and 18) - hence the subscripts "N" and "D" has been left out. This relation joins important parameters of the growth process - the slope of liquidus surface $k_{i}$ with respect to an arbitrary dilute $i$-component, the supersaturation $\Delta C_{i}^{L^{*}}$ with respect to the same component, the time constant $\tau_{i}^{(S u \text {. })}$ with respect to $i$-component again and the cooling rate $v_{T}$. The relation can be applied to techniques of supercooling and equilibrium cooling, at which the temperature decrease is in use: $v_{T} \neq 0$.

It is worth to point out that in dislocation mechanism of growth two time constants, $\tau_{i}^{(S u .)}$ and $\tau_{00 i . D}$ are revealed. $\tau_{i}^{(S u .)}$ concerns the transient regime of growth - it provides the rate of transition of the system from the onset of the process, where a kinetic limited growth takes place, to reaching of the stationary growth regime, where the process is dominated by the diffusion mass transport. The type of process concerning the second time constant $\tau_{00 i . D}$ will be defined later in the text.

\subsection{Supersaturation, growth rate and growth layer thickness versus time: dependences $\Delta C_{i}^{L}(t), v_{g r}(t)$ and $d(t)$}

Equations 8-16 and 18-26 allow the investigation of the dependences of the supersaturations, the growth rates and the growth layer thicknesses at both crystallization mechanisms for different growth techniques on the duration of growth $t$. With this aim equations have been rewritten by the use of generalized variables. For the supercooling technique the 
variables are: the relative growth rate $y^{(S u .)}=v_{g r}^{(S u .)} / v_{g r}^{*}$, the relative supersaturation $x_{i}^{(S u .)}=\Delta C_{i}^{L(S u .)^{*}} / \Delta C_{i .0}^{L}$, the dimensionless time $\theta_{i}^{(S u .)}=t / \tau_{i}^{(S u .)}$ and the relative layer thickness $D^{(S u .)}=d^{(S u .)} / v_{g r}^{*} \tau_{i}^{(S u .)}$.

In case of equilibrium cooling the corresponding relations are: $y^{(E q .)}=v_{g r}^{(E q \cdot)} / v_{g r}^{*}$, $\theta_{i}^{(E q .)}=t / \tau_{i}^{(E q .)}$ and $D^{(E q .)}=d^{(E q .)} / v_{g r}^{*} \tau_{i}^{(E q .)}$. The subscripts "N" and "D" are missing again because of the similarity of relations.

In step cooling technique the relations are: $y_{N}^{(S t .)}=v_{g r N}^{(S t .)} / v_{g r 0 N}, \theta_{i . N}^{(S t .)}=t / \tau_{i . N}^{(S t .)}$, $D_{N}^{(S t .)}=d_{N}^{(S t .)} / v_{g r 0 . N} \tau_{i N}^{(S t .)}$ at normal mechanism and $y_{D}^{(S t .)}=v_{g r D}^{(S t .)} / v_{g r 0 D}, \theta_{i . D}^{(S t .)}=t / \tau_{00 i . D}$, $D_{D}^{(S t .)}=d_{D}^{(S t .)} / v_{g r 0} \tau_{00 i . D}$, at dislocation one.

Figure 1 shows the dependences $y_{N}^{(S u .)}=v_{g r N}^{(S u .)} / v_{g r N}^{*}$ and $y_{D}^{(S u .)}=v_{g r D}^{(S u .)} / v_{g r D}^{*}$ on the dimensionless time $\theta_{i}^{(S u .)}$ for both growth mechanisms. Behavior of the system in both cases is similar - at the beginning of the process (at $\theta_{i}^{(S u .)}<1$ or $t<\tau_{i}^{(S u .)}$ ) the growth rate is very high due to the initial supercooling. A very sharp decrease of the growth rate is observed because the system tends to achieve stationary conditions. At dislocation mechanism the sharpness of the decrease is significantly greater. With time the process tends to grow stationary and the growth rate becomes almost constant - at dislocation mechanism this occurs faster.

A.

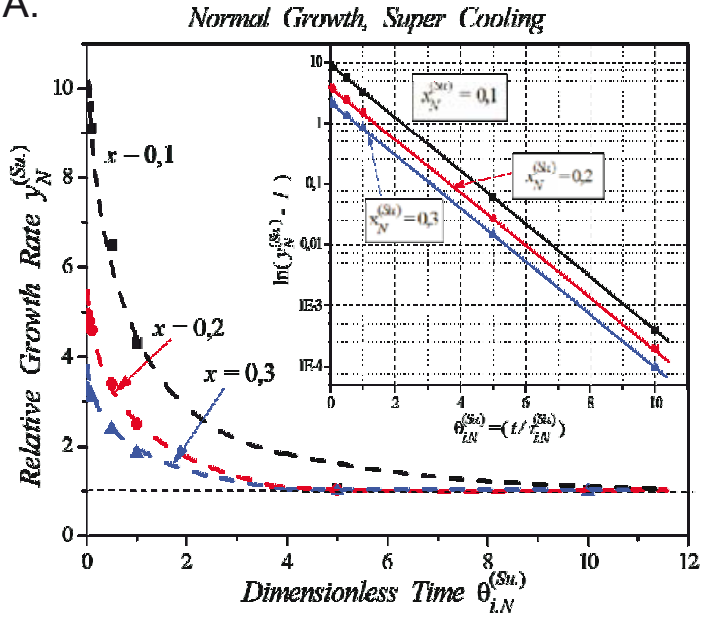

B.

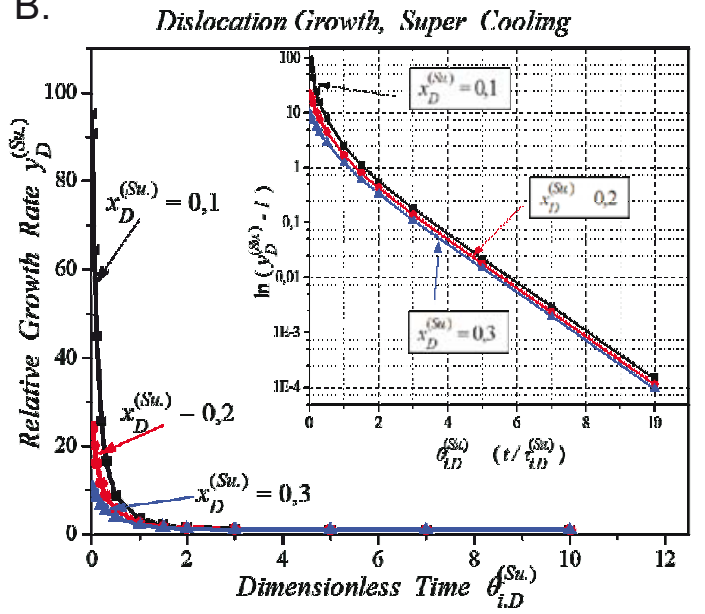

Figure 1: Dependences $y_{N}^{(S u .)}\left(\theta_{i . N}^{(S u .)}\right)$ and $y_{D}^{(S u .)}\left(\theta_{i . D}^{(S u .)}\right)$. The inset shows the same curves in coordinates $\ln \left(y^{(S u .)}-1\right) \Leftrightarrow \theta_{i}^{(S u .)}$.

The similarity of both curves in linear coordinates is obvious. This circumstance hinders both mechanisms to be recognized clearly. On the inset of both figures the same relative growth rates, $y_{N}^{(S u .)}$ and $y_{D}^{(S u .)}$ are shown in coordinates $\ln \left(y^{(S u .)}-1\right) \Leftrightarrow \theta_{i}^{(S u .)}$. In this case there is an obvious difference between the curves of both mechanisms. At normal growth the dependence $\ln \left(y_{N}^{(S u .)}-1\right) \Leftrightarrow \theta_{i . N}^{(S u .)}$ is linear, but at dislocation one it is nonlinear. Just at the beginning (at $\theta_{i}^{(S u .)} \approx 1$ ) a nonlinearity is observed which becomes a straight line at great duration of the process $\left(\theta_{i}^{(S u .)}>>1\right)$. This difference allows a reliable method for 
distinguishing the two mechanisms in case of the supercooling technique.

The same situation occurs in case of equilibrium and step cooling techniques (fig. 2).
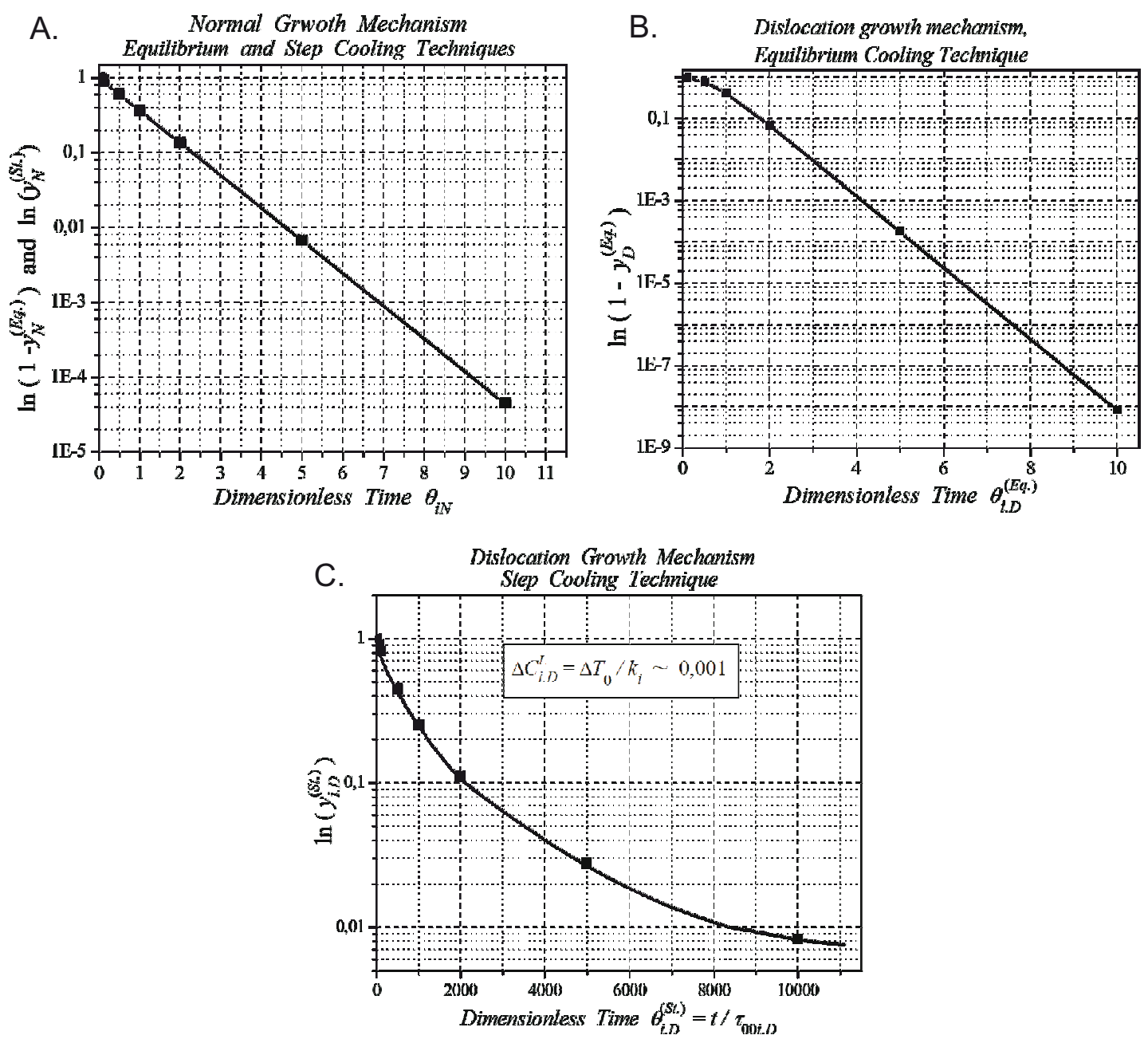

Figure 2: Relative growth rate at normal and dislocation mechanisms of growth versus dimensionless time: A. Relative growth rates $y_{N}^{(E q .)}\left(\theta_{i . N}^{(E q .)}\right)$ and $y_{N}^{(S t .)}\left(\theta_{i . N}^{(S t .)}\right)$ in the corresponding coordinates $\ln \left(1-y_{N}^{(E q .)}\right) \Leftrightarrow \theta_{i . N}^{(E q .)}$ and $\ln \left(y_{N}^{\left(S S_{t}\right)}\right) \Leftrightarrow \theta_{i . N}^{(S t .)}$ for normal mechanism at equilibrium and step cooling techniques - both dependences coincide with each other; B. Relative growth rate $y_{D}^{(E q .)}\left(\theta_{i . D}^{(E q .)}\right)$ in coordinates $\ln \left(1-y_{D}^{(E q .)}\right) \Leftrightarrow \theta_{i . D}^{(E q .)}$ at dislocation growth and equilibrium cooling; C. Relative growth rate $y_{D}^{(S t .)}\left(\theta_{i . D}^{(S t .)}\right)$ in coordinates $\ln \left(y_{D}^{(S t .)}\right) \Leftrightarrow \theta_{i . D}^{(S t .)}$ at dislocation growth and step cooling.

At equilibrium cooling both relative velocities $y_{N}^{(E q .)}\left(\theta_{i . N}^{(E q .)}\right)$ (fig. $\left.2 \mathrm{~A}\right)$ and $y_{D}^{(E q .)}\left(\theta_{i . D}^{(E q .)}\right)$ (fig. 2 B) in coordinates $\ln \left(1-y^{(E q .)}\right) \Leftrightarrow \theta_{i}^{(E q .)}$ demonstrate different behavior - at normal mechanism the exponential function from eq. 12 is a straight line but in dislocation one eq. 22 provides a nonlinear relation. In case of step cooling the curves of relative growth rates $y_{N}^{(S t .)}\left(\theta_{i . N}^{(S t .)}\right)\left(\right.$ fig. 2 A) and $y_{D}^{(S t .)}\left(\theta_{i . D}^{(S t .)}\right)$ (fig. 2 C) in coordinates $\ln \left(y^{(S t .)}\right) \Leftrightarrow \theta_{i}^{(S t .)}$ 
demonstrate the same behavior - for the normal mechanism (eq. 13) the dependence is linear but for the dislocation one it is nonlinear (eq. 23).

At normal mechanism the curves of equilibrium cooling and step cooling coincide with each other. This is the reason why they are presented by a common curve on fig. 2 A. This coincidence does not mean that the growth rate at both techniques will be the same. Along the ordinate axis the values of the function $\ln \left(1-y_{N}^{(E q .)}\right)$ are presented for equilibrium cooling and the values of $\ln \left(y^{(S t .)}\right)$ for step cooling. At equilibrium cooling the relative growth rate $y_{N}^{(E q .)}$ is given by the ratio between the real growth rate $v_{g r N}^{(E q .)}(t)$ and the stationary growth rate $v_{g r N}^{(E q .)^{*}}, y_{N}^{(E q .)}=v_{g r N}^{(E q .)} / v_{g r N}^{(E q .)^{*}}$. At step cooling the relative growth rate $y_{N}^{(S t .)}$ is the ratio between the real growth rate $v_{g r N}^{(S t .)}$ and the initial growth rate $v_{g r 0 N}, y_{N}^{(S t .)}=v_{g r N}^{(S t)} / v_{g r 0 N}$. Along the $x$-axis the values of the dimensionless time are given by the ratio $\theta_{i . N}^{(E q .)}=t / \tau_{i . N}^{(E q .)}$ in case of equilibrium cooling, and by $\theta_{i . .}^{(S t .)}=t / \tau_{i . N}^{(S t .)}$ in case of step cooling. Both curves can really coincide with each other if the values of both time constants $\tau_{i . N}^{(E q .)}$ and $\tau_{i . N}^{(S t .)}$ are equal but as a rule it is not the case - they are different.

The coordinates $\ln \left(y^{(S u .)}-1\right) \Leftrightarrow \theta_{i}^{(S u .)}, \ln \left(1-y^{(E q .)}\right) \Leftrightarrow \theta_{i}^{(E q .)}$ and $\ln \left(y^{(S t .)}\right) \Leftrightarrow \theta_{i}^{(S t .)}$ in case of supercooling, equilibrium cooling and step cooling, respectively, reveal the exponential character of the dependences for the normal mechanism of growth. The corresponding equations 11, 12 and 13 in the respective coordinates are always presented by straight lines - the linear dependence in these coordinates means that the growth rate $v_{g r . N}(t)$ depends on the time exponentially. In the same coordinates equations 21,22 and 23 for dislocation mechanism of growth are always nonlinear. This is the main difference by which the two mechanisms can be distinguished.

For the supercooling technique the thickness of the layer increases continuously with the duration of growth (fig. $3 \mathrm{~A}$ and $\mathrm{B}$ ) - at the beginning (at $\theta_{i}^{(\mathrm{Su} .)}<1$ ) it occurs nonlinearly, when a kinetically limited growth takes place. With increasing duration of growth $\left(\theta_{i}^{(S u .)}>1\right)$ the layer thickness increases linearly due to the stationary regime of growth - the growth rate is constant $\left(v_{g r}(t)=v_{g r}^{*}\right)$. For both mechanisms the curves are similar - the only difference occurs during the transient regime where the kinetics dominates - at normal mechanism the duration of the kinetic regime is two-three times longer.

These curves reveal the values of the stationary growth rate $v_{g r}^{*}$ and the time constant $\tau_{i}^{(S u .)}$. The slope $\alpha$ of the linear part of the curves gives the value of the stationary growth rate $v_{g r}^{*}$ which is reasonable since the layer thickness increases linearly with time when the growth rate is constant, as in the case of stationary growth. The point of intersection $D^{(S u .)^{* *}}$ (or $d^{(S u .)^{* *}}$, respectively) with the ordinate axis of the interpolated linear part of the curve allows the determination of the time constant $\tau_{0}^{(S u .)}$ by the expression:

$$
\tau_{0}^{(S u .)}=\frac{1}{\chi}-\frac{d^{(S u .)^{* *}}}{v_{g r}^{*}}
$$

where $\chi=v_{T} / \Delta T_{0}$ (eq. 29), $\Delta T_{0}$ is the initial supercooling and $D^{(S u .)^{* *}}=d^{(S u .)^{* *}} / v_{g r}^{*} \tau_{i}^{(S u .)}$ is the relative layer thickness at the point of intersection. Parameter $\chi$ is preliminary known, defining $v_{T}$ and $\Delta T_{0}$ before the experiment. Equation 27 is valid for both mechanisms of growth. In this equation we have assumed that the time constant $\tau_{i}^{(S u \text {. })}$ is the same for all components - the transient process begins and ends for all components 
simultaneously: $\tau_{1}^{(S u .)}=\tau_{2}^{(S u .)}=\ldots=\tau_{n_{0}}^{(S u .)}=\tau_{0}^{(S u .)}$.

At equal parameters $x_{i . N}^{(S u .)}$ and $x_{i . D}^{(S u .)}$ the coordinates $D^{(S u .)^{* *}}$ (or $d^{(S u .)^{* *}}$, respectively) of both mechanisms are equal: $D_{N}^{(S u .)^{* *}} \equiv D_{D}^{(S u .)^{* *}}$ and $d_{N}^{(S u .)^{* *}} \equiv d_{D}^{(S u .)^{* *}}$ (fig. 3). In this case the time constant of normal growth must be equal to that of the dislocation growth (it follows from equation 27 and is shown on fig. 3).

A.

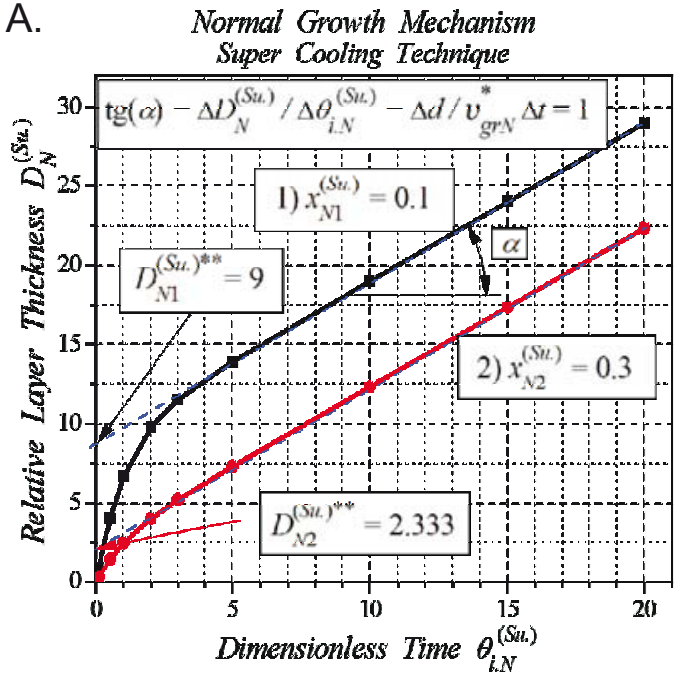

B.

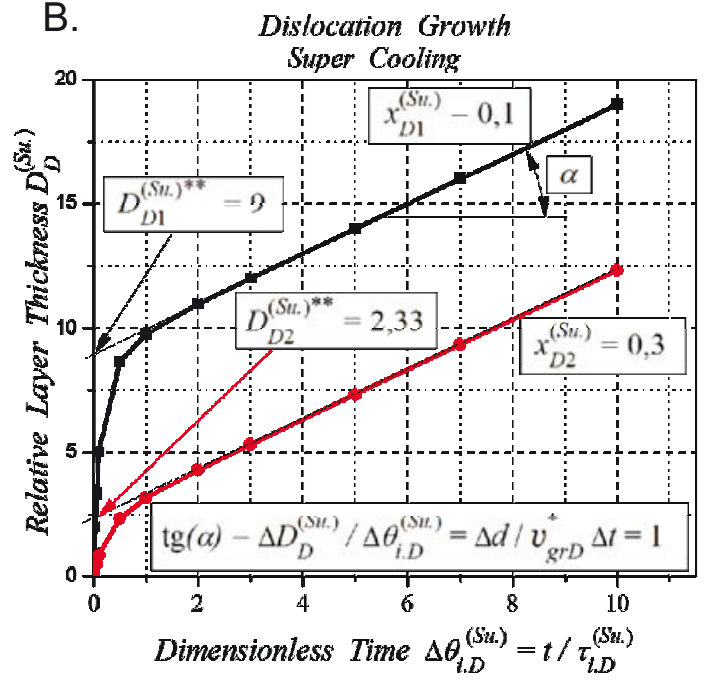

Figure 3: Layer thickness versus growth time for both mechanisms and supercooling technique.

With the aid of the stationary growth rate $v_{g r}^{*}$ and the time constant $\tau_{0}^{(S u .)}$ one may determine all other parameters of the growth process - the relative supersaturation $x_{i}^{(S u .)}$, the kinetic coefficient $\mu_{i}$, the stationary supercooling $\Delta T^{*}$, the initial growth rate $v_{g r .0}$.

In case of equilibrium cooling (figure 4 ) for both mechanisms of growth the layer thickness increases with time continuously. Initially $\left(\right.$ at $\left.\theta_{0}^{(S u .)}<1\right)$ the dependence is nonlinear but with increasing duration of growth it becomes linear, which corresponds to the beginning of the stationary growth regime. The slope of the linear part, given by the angle $\alpha$ (fig. 4), provides the corresponding growth rate $v_{g r}^{*}$ at stationary growth conditions (at $\left.\theta_{0}^{(S u .)}>1\right)$. The point of intersection $\theta_{0}^{(E q \cdot)^{* *}}=t^{(E q \cdot)^{* *}} / \tau_{0}^{(E q .)}=1 \longrightarrow t^{(E q .)^{* *}}=\tau_{0}^{(E q .)}$, with the $x$-axis of the prolongation of the linear part of the curve gives the value of the respective time constant $\tau_{0}^{(E q .)}$ (fig. $4 \mathrm{~A}$ - curve 1 and B - curve $\left.D_{D}^{(E q .)}\left(\theta_{0 . D}^{(E q .)}\right)\right)$.

In case of step cooling in both mechanisms (fig. 4 A curve 2 and B curve $D^{(S t .)}\left(\theta_{0}^{(S t .)}\right)$ ) layer thickness increases with time initially and then remains constant - it means that the growth process is interrupted because the initial supercooling has ended.

The dependence of the relative growth rates $y_{D}^{(E q .)}\left(\theta_{0 . D}^{(E q .)}\right)$ and $y_{D}^{(S t .)}\left(\theta_{0 . D}^{(S t .)}\right)$ on time is presented on figures $4 \mathrm{~B}$ and $\mathrm{C}$ for the dislocation mechanism for both techniques (equilibrium and step cooling). At equilibrium cooling the growth rate increases with time continuously and after reaching its stationary value remains constant - the stationary growth rate is the maximal value of the growth rate in case of equilibrium cooling. In case of step cooling, from the beginning the growth rate decreases continuously tending to zero because the initial supercooling has ended. 
A.

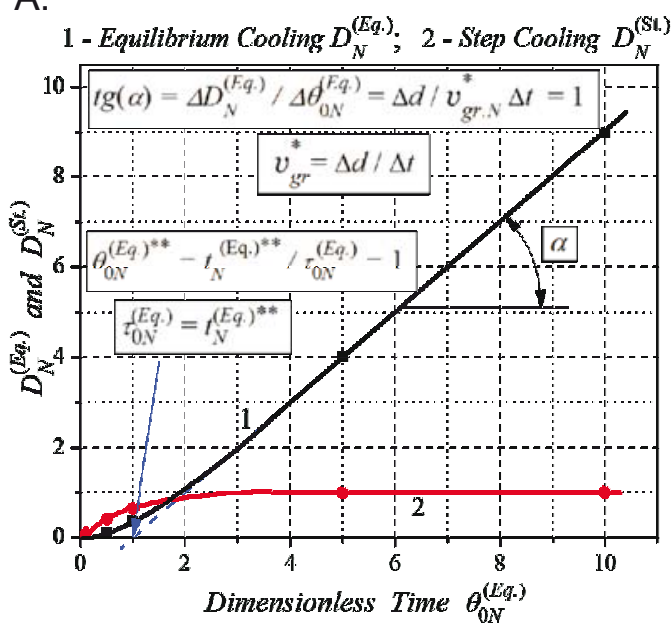

B.

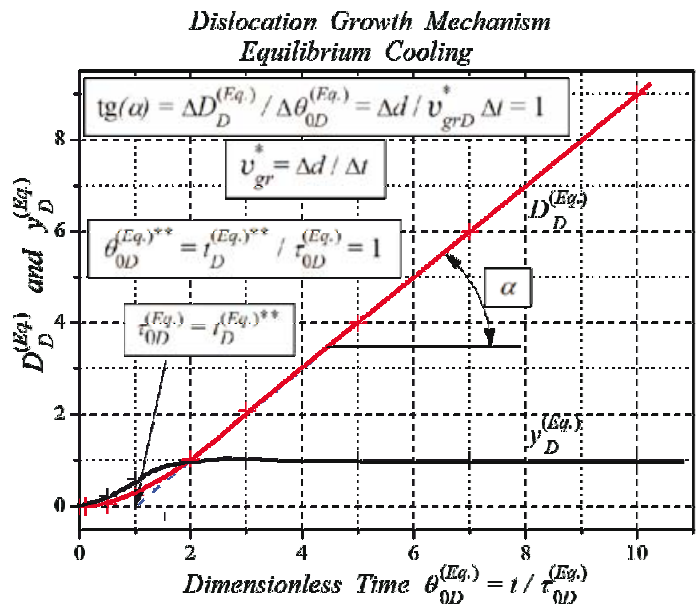

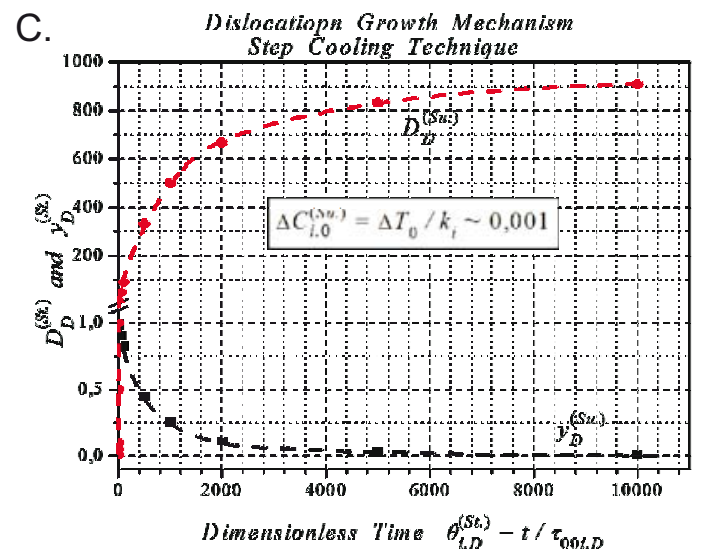

Figure 4: Dependences $D^{(E q .)}\left(\theta_{i}^{(E q .)}\right)$ and $D^{(S t .)}\left(\theta_{i}^{(S t .)}\right)$ at normal and dislocation growth: A. Normal growth mechanism at equilibrium cooling and step cooling, B. Dislocation growth mechanism at equilibrium cooling, C. Dislocation growth at step cooling.

\subsection{Parameters of the process}

Lets return to the relation observed in equations 7 and 18, which is common for both mechanisms of crystallizations,:

$$
v_{T} \tau_{0}=k_{i} \Delta C_{i}^{L^{*}}=\Delta T^{*}
$$

The subscripts "N" and "D" are omitted because the relation is valid for both mechanisms. This relation connects important parameters of the process - the cooling rate $v_{T}$, the time constant $\tau_{0}$, the slope of liquidus surface $k_{i}$ with respect to each dilute $i$-component, the stationary value of superstauration $\Delta C_{i}^{L^{*}}$ with respect to the same component and the stationary supercooling $\Delta T^{*}$. The greater the time constant is, the slower is the transition of the system towards the stationary growth regime and vice versa.

In case of normal mechanism (eq. 7) the time constant $\tau_{0 N}$ depends on the process temperature $T_{0}$, the solubility $\left(C_{i}^{L}\right.$ and $\left.C_{k}^{L}\right)$, the area of the growth layer $S_{0}$, the mass 
of the melt $m_{k}^{L}$ (the mass of the basic component), the slope of liquidus surface and the kinetic coefficient $\mu_{i . N}$ for the normal mechanism and it does not depend on the cooling rate $v_{T}$.

In case of dislocation mechanism the time constant $\tau_{0 D}^{(S u .)}$ depends on the same parameters mentioned above and, additionally, on the cooling rate $v_{T}$ - it decreases with enhanced cooling: $\tau_{0 D}^{(S u .)}=\left(k_{i} / v_{T} G_{i . D}\right)^{1 / 2}$ (eq. 18). The change of the stationary supersaturation with the cooling rate occurs by another manner - with enhancing cooling it increases: $\Delta C_{i . D}^{L} \equiv \Delta C_{i . D}^{L^{*}}=\left(v_{T} / k_{i} G_{i . D}\right)^{1 / 2}$ (eq. 18).

Using eq. 28 one obtains relations concerning other parameters of the process:

- relative supersaturations $x_{N}^{(S u .)}$ and $x_{D}^{(S u .)}$ at arbitrary value of the cooling rate $v_{T 1}$ (for normal mechanism) and $v_{T 2}$ (for dislocation mechanism) using eq. 28

$$
\begin{aligned}
x_{N}^{(S u .)} & =\frac{\Delta C_{m .1}^{L^{*}}}{\Delta C_{m .0}^{L}}=\frac{\Delta T_{1}^{*}}{\Delta T_{0}}=\frac{v_{g r 1}^{*}}{v_{g r .0 N}}=\frac{v_{T 1} \tau_{0 N}}{\Delta T_{0}}=\chi_{1} \tau_{0 N} \\
x_{D}^{(S u .)} & =\frac{\Delta C_{m .2}^{L^{*}}}{\Delta C_{m .0}^{L}}=\frac{\Delta T_{2}^{*}}{\Delta T_{0}}=\sqrt{\frac{v_{g r 2}^{*}}{v_{g r .0 D}}}=\frac{v_{T 2} \tau_{0 D}}{\Delta T_{0}}=\chi_{2} \tau_{0 D} \\
\chi_{1} & =\frac{x_{N}^{(\text {Su. })}}{\tau_{0 N}}=\frac{v_{T 1}}{\Delta T_{0}} ; \quad \chi_{2}=\frac{x_{D}^{(S u .)}}{\tau_{0 D}}=\frac{v_{T 2}}{\Delta T_{0}}
\end{aligned}
$$

- kinetic coefficients $\mu_{m . N}$ and $\mu_{m . D}$ by eq. 28

$$
\mu_{i . N}=\frac{v_{g r 1}^{*}}{v_{T 1} \tau_{0 N}}, \quad \mu_{i . D}=\frac{v_{g r 2}^{*}}{\left(v_{T 2} \tau_{0 D}\right)^{2}}
$$

- initial growth rates $v_{g r 0 N}$ and $v_{g r 0 D}$ by eq. 29

$$
v_{g r 0 N}=\frac{v_{g r 1}^{*}}{x_{N}^{(S u .)}}, \quad v_{g r 0 D}=\frac{v_{g r 2}^{*}}{\left(x_{D}^{(S u .)}\right)^{2}}
$$

These relations allow determining almost all important parameters of the growth process. It is worth to point out that at equal cooling rates $v_{T 1}$ and $v_{T 2}, v_{T 1}=v_{T 2}$, taking into account eq. 5, one obtains that both relative supersaturations from eq. 29 are equal, $x_{N}^{(S u .)}=x_{D}^{(S u .)}$, and many other parameters become equal too: $\chi_{1}=\chi_{2}, \tau_{0 N}=\tau_{0 D}$, $v_{g r .1}^{*}=v_{g r .2}^{*}, \Delta T_{1}^{*}=\Delta T_{2}^{*}$. Only the kinetic coefficients $\mu_{i . N}$ and $\mu_{i . D}$, and the initial growth rates $v_{g r 01}$ and $v_{g r 02}$ remain different.

\section{Experiment: Liquid Phase Epitaxy of GaAs}

Experimental results are published in [39]. GaAs LPE layers are grown using graphite boat of type "pusher", shown on fig. 5. The boat has been developed aiming the growth of thin epitaxial layers. Its construction ensures the thickness of the liquid phase over the substrate to be very thin - less than $2 \mathrm{~mm}$.

The liquid phase is sprayed over the substrate by the pusher, after the previous melt has been pushed out. It occurs very fast within a very short time interval (less than $1 \mathrm{~s}$ ) and any mixing of both melts (the present and the previous) does not take place. The spraying of the melts is a precondition for the good wetting of the substrate by the melt - the layers 
grow uniformly over the whole surface. After finishing the experiment the substrate was cut off. By wet etching the layers interfaces have been revealed and by optical microscopy the grown layer thickness was measured.

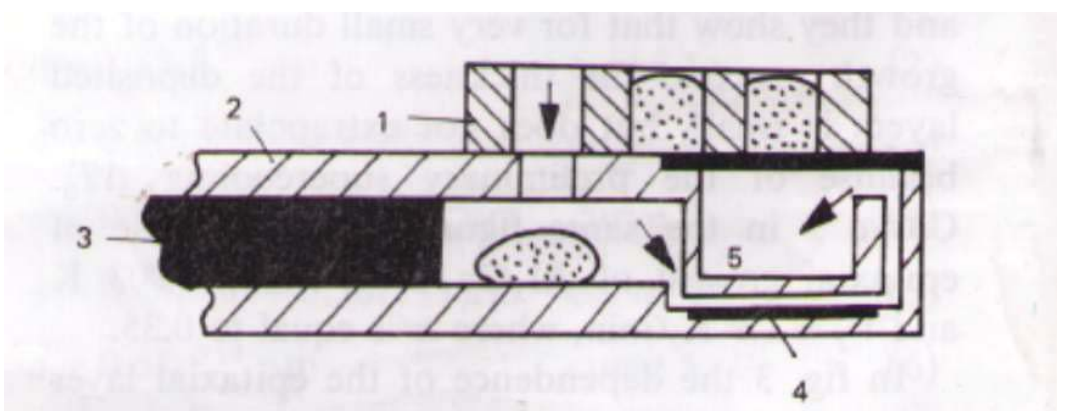

Figure 5: The "pusher" graphite boat consists of: 1) a slider with the melts; 2) body of the boat; 3) pusher; 4) substrate 5) volume for the used melts. By arrows the motion of the melts in the boat is given [39].

In this way four curves have been established, grown at different conditions - different growth temperature $T_{0}$, cooling rate $v_{T}$, initial supercooling $\Delta T_{0}$. In case of curve 1 the layers were grown at $T_{0}=1053 \mathrm{~K}$, by cooling rate $v_{T}=1.9 \mathrm{~K} / \mathrm{min}$ and initial supercooling $\Delta T_{0}=5 \mathrm{~K}$. Because of the great dispersion in the experiments it has not been considered. Curves 2, 3 and 4 are presented on fig. $6 \mathrm{~A}, \mathrm{~B}$ and C. Because a supercooling technique has been used, the curves from fig. 3 are to be taken into account.

All three curves repeat sufficiently well the shape of the curves $D(\theta)$ presented on fig. 3 . The linear part of the curve reveals the stationary growth regime. The slope of the curves provides the value of the stationary growth rate. The prolongation of the linear part toward the ordinate axis provides the value of the coordinate of the point of intersection $d^{(S u .)^{* *}}$ and with the aid of them, using equation 27 , the time constant of the process $\tau_{0}$ has been determined. The growth conditions and the corresponding values of $v_{g r}^{*}$ and $d^{(S u .)^{* *}}$ are given on fig. 6 .

We will further discuss curve 2 only - curves 3 and 4 are similar.

By the established values of $v_{g r}^{*}$ and $\tau_{0}$ and by the initially known cooling rate $v_{T}$ and initial supercooling $\Delta T_{0}$, using equations 28-31 we have determined the main parameters of the process. The obtained results are given in Table 1 for each curve and for both mechanisms of growth. By the already determined $v_{g r}^{*}$ and $\tau_{0}$ one may evaluate which of the two crystallization mechanisms reproduces the experimental results better.

With this aim, using equations 14 and 24, the time dependence of the grown layer thickness $d_{D}^{(S u .)}(t)$ and $d_{N}^{(S u .)}(t)$ for both mechanisms has been determined. The necessary values of parameters $v_{g r}^{*}, \tau_{0}$ and $x$ are available in Table 1 . The results are presented on figure 7. Curve $d_{D}^{(S u .)}(t)$ reproduces the experimental results better than the curve $d_{N}^{(S u .)}(t)$. It is observed in all curves 2, 3 and 4 .

Using equations 14 and 24, concerning the increasing with the duration of the growth layer thickness, $d_{D}^{(S u .)}(t)$ and $d_{N}^{(S u .)}(t)$, the averaged value of the growth rate at normal and dislocation mechanisms of growth has been determined: $v_{g r}^{\text {aver. }}(t)=d^{(S u .)}(t) / t$. The corresponding dependence $v_{g r}^{\text {aver. }}(t)$ is presented on fig. 8. Averaged values of the growth 
rate, experimentally determined by the data of fig. $6 \mathrm{~A}$, are presented on figure 8 . Obviously, equation 24 concerning dislocation mechanism of growth reproduces much better the experimental results for all three curves 2, 3 and 4. One may conclude that in our experiments the layers are grown by dislocation mechanism of crystallization.
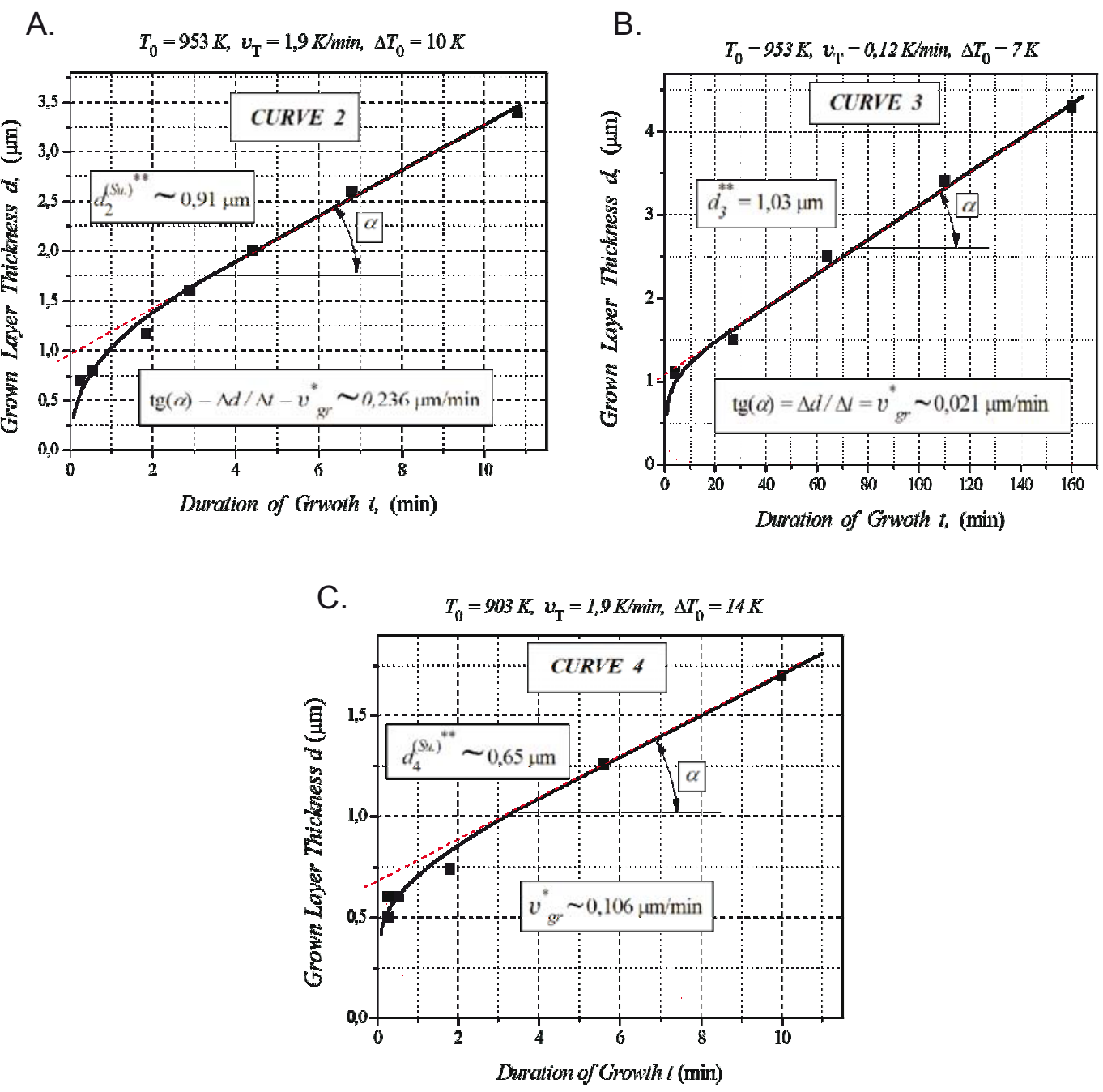

Figure 6: Grown layer thickness versus duration of growth $d^{(S u .)}(t)$.

One must point out that in the experiments usually the averaged growth rate is observed - it is the thickness of the grown layer divided by the duration of its growth: $v_{g r}^{\text {aver. }}(t)=$ $d^{(S u .)}(t) / t$. Equations, obtained above (equations 11-13 and 21-23), concerning growth rates $v_{N}(t)$ and $v_{D}(t)$ at the different techniques, provide the value of the actual growth rate: $v_{g r}^{a c t .}\left(t^{\prime}\right)=\Delta d\left(t^{\prime}\right) / \Delta t$ - this is the growth rate at an arbitrary time $t^{\prime}$, where $\Delta t$ is a very small time interval around the time $t^{\prime}$. The difference between both values of the growth rate (the averaged and the actual) may reach $200-300 \%$. Using the values of Table 1 and equations 11 and 21 (valid for the actual values $v_{g r . N}^{a c t .(S u .)}(t)$ and $v_{g r . D}^{a c t .(S u .)}(t)$ ) the dependence of actual growth rate versus time has been defined and presented on fig. 9. For comparison, the curve $v_{g r}^{a v e r} .(t)$ is presented too. The averaged value of the growth 
rate is 2-3 times larger than the actual one.

Table 1: Process parameters of curves 2, 3 and 4 in case of normal and screw dislocation mechanisms of crystallization.

\begin{tabular}{c|cc|cc|cc}
\hline \hline & \multicolumn{2}{|c|}{ Curve 2} & \multicolumn{2}{c|}{ Curve 3 } & \multicolumn{2}{c}{ Curve 4 } \\
\cline { 2 - 7 } & $\begin{array}{c}\text { Normal } \\
\text { mech. }\end{array}$ & $\begin{array}{c}\text { Disloc. } \\
\text { mech. }\end{array}$ & $\begin{array}{c}\text { Normal } \\
\text { mech. }\end{array}$ & $\begin{array}{c}\text { Disloc. } \\
\text { mech. }\end{array}$ & $\begin{array}{c}\text { Normal } \\
\text { mech. }\end{array}$ & $\begin{array}{c}\text { Disloc. } \\
\text { mech. }\end{array}$ \\
\hline$\tau(\mathrm{min})$ & $\approx 1.38$ & $\approx 1.38$ & $\approx 9.30$ & $\approx 9.30$ & $\approx 1.24$ & $\approx 1.24$ \\
$x$ & $\approx 0.260$ & $\approx 0.260$ & $\approx 0.159$ & $\approx 0.159$ & $\approx 0.168$ & $\approx 0.168$ \\
$v_{g r}^{*}(\mu \mathrm{m} / \mathrm{min})$ & $\approx 0.236$ & $\approx 0.236$ & $\approx 0.021$ & $\approx 0.021$ & $\approx 0.106$ & $\approx 0.106$ \\
$\chi\left(\mathrm{min}^{-1}\right)$ & $\approx 0.190$ & $\approx 0.190$ & $\approx 0.017$ & $\approx 0.017$ & $\approx 0.136$ & $\approx 0.136$ \\
$\Delta T^{*}(\mathrm{~K})$ & $\approx 2.60$ & $\approx 2.60$ & $\approx 1.10$ & $\approx 1.10$ & $\approx 2.36$ & $\approx 2.36$ \\
$v_{g r 0}(\mu \mathrm{m} / \mathrm{min})$ & $\approx 0.91$ & $\approx 3.50$ & $\approx 0.13$ & $\approx 0.83$ & $\approx 0.64$ & $\approx 3.20$ \\
$\mu$ & $\approx 15.0$ & $\approx 5.8$ & $\approx 3.2$ & $\approx 2.9$ & $\approx 7.5$ & $\approx 9.0$ \\
$\left(10^{-8} \mathrm{~cm} / \mathrm{s} \cdot \mathrm{K}\right)$ & & & & & & \\
\hline \hline
\end{tabular}

In coordinates $\ln \left(y^{(S u .)}-1\right) \Leftrightarrow t$, concerning the supercooling technique, the experimental results are presented on the inset. It has been already established (fig. 1) that if at supercooling technique a normal growth mechanism takes place the curve $y^{(S u}$.) $(t)$ in the coordinates $\ln \left(y^{(S u .)}-1\right) \Leftrightarrow t$ must be a straight line (fig.1 A). If at the same technique a dislocation growth occurs then in the same coordinates the dependence $\left.y^{(S u} \cdot\right)(t)$ must be nonlinear (fig. 1 B). The curve on the inset of fig. 9 is obviously nonlinear and agrees well with the curve on the inset of fig. $1 \mathrm{~B}$. The same nonlinearity is observed on curves 3 and 4. It confirms the conclusion made above that in our experiments dislocation mechanism of crystallization takes place.

\section{Super Cooling Technique}

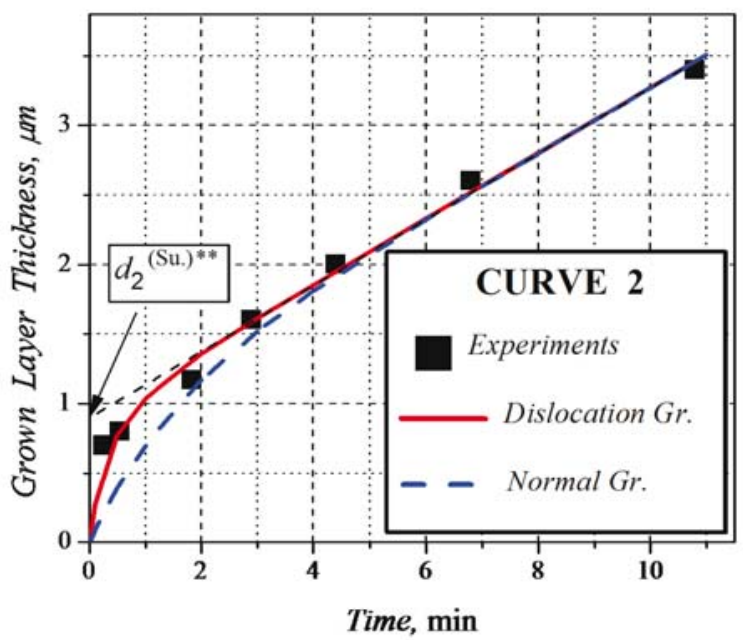

Figure 7: Experimental points $d^{(S u .)}(t)$ versus curves, obtained by equations 14 and 24 concerning normal and dislocation mechanisms of growth. 


\section{Super Cooling Technique}

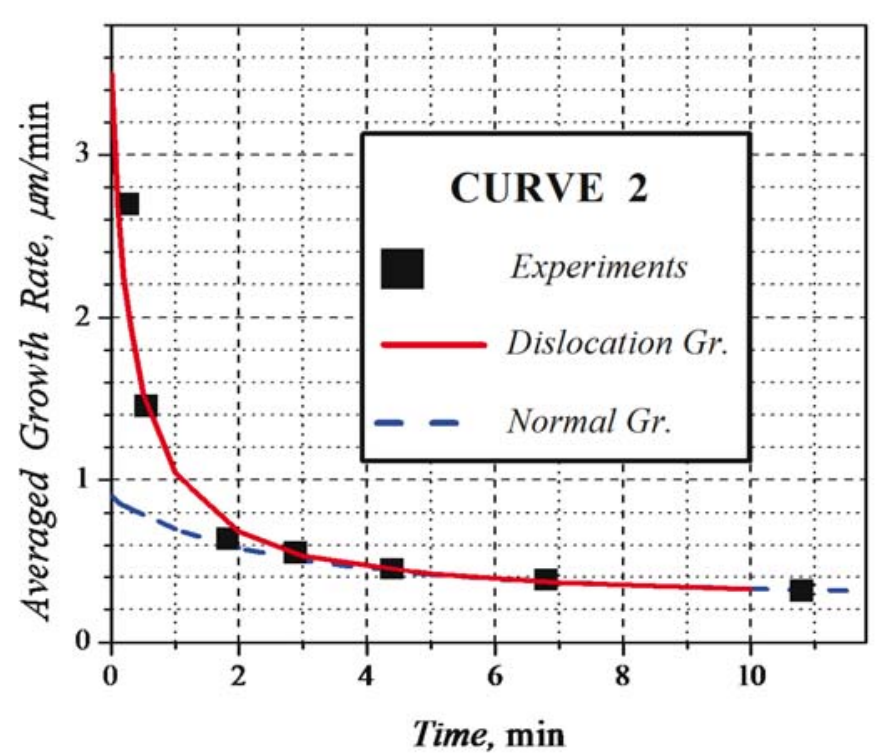

Figure 8: Averaged growth rate versus time - experiments and reproduction of the same curve by both mechanisms.

\section{CURVE 2}

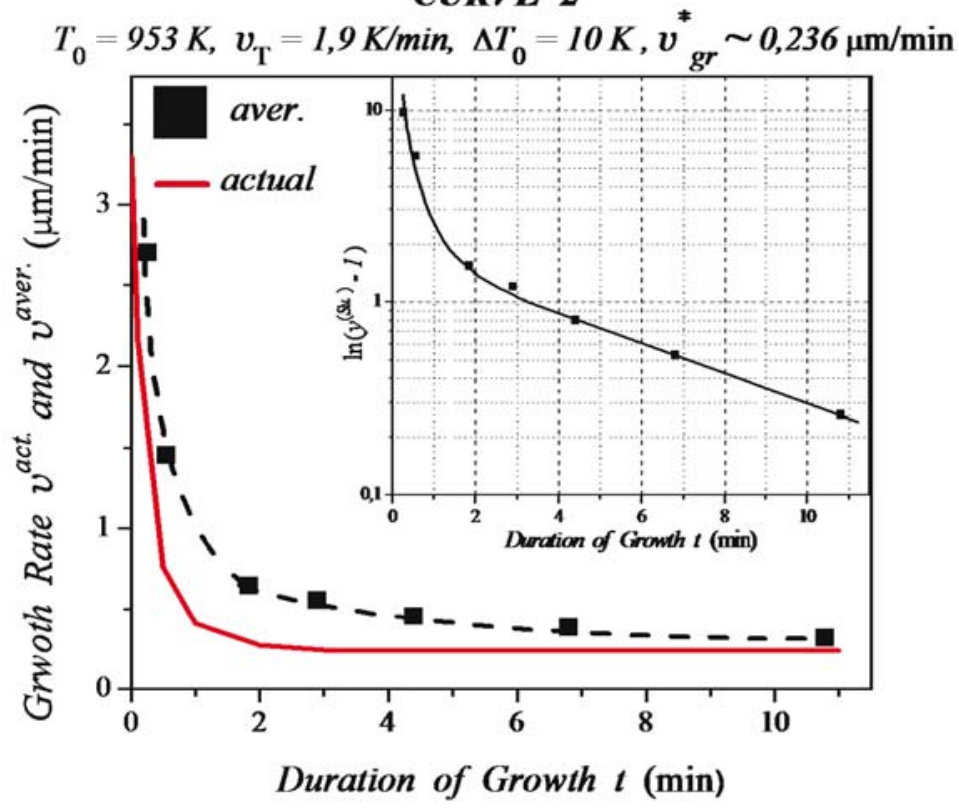

Figure 9: The actual and the averaged growth rates. 


\section{Discussion}

The presented approach concerning the liquid phase epitaxial growth allows the definision of the main process parameters - stationary growth rate $v_{g r}^{*}$, stationary supercooling $\Delta T^{*}$, initial growth rate $v_{g r .0}^{(S u .)}$, time constant $\tau_{0}^{(S u .)}$, kinetic coefficient $\mu$, relative supercooling $x^{(S u .)}$. The quantity $x^{(S u .)}$ is valid for the supercooling technique only: $x^{(S u .)}=\Delta C_{i}^{*} / \Delta C_{i .0}^{L}$. For other techniques there is no such quantity - for the equilibrium cooling the initial supersaturation is zero $\Delta C_{i .0}^{L}=0$ and for step cooling the stationary supersaturation is zero $\Delta C_{i}^{*}=0$. The supercooling technique is that LPE technology, which uses all the tools of the liquid phase growth - the initial supercooling and the temperature decrease. This is the reason why our attention is drawn to this technique.

The presented approach allows the determination of the crystal growth mechanism. The relative growth rate $y$ presented in the corresponding coordinates, which are specific for each growth technique mentioned above, reveals the crystallization mechanism. At normal mechanism in these coordinates the relative growth rate is always a straight line but at dislocation mechanism it is always nonlinear. Using the values of the averaged growth rate, determined by the experiments, the relative growth rate $y$ versus the duration of growth $t$ was defined (inset of fig. 9) - the curve is in a good agreement with the curve presented on the inset of fig. $1 \mathrm{~B}$. Both curves differ in two points:

- On fig. $1 \mathrm{~B}$ the relative growth rate $y$ as function of the dimensionless time $\theta$ is presented. On the other hand, as can be seen on fig. 9, the relative growth rate depends on the real time $t$. Both quantities, $\theta$ and $t$, differ by the time constant $\tau$ and this constant can't affect the type of the dependence - if the dependence is linear it will remain linear and vice versa.

- The curve on fig. $1 \mathrm{~B}$ represents the actual value of the growth rate, yet the curve on fig. 9 represents the averaged value. In this situation one may expect a qualitative agreement between theory and experiments. It is almost impossible to obtain a quantitative agreement because experiments provide the averaged value of the growth rate and not the actual value.

The similarity between figures $1 \mathrm{~B}$ and 9 can be regarded as a confirmation of the behaviour of the process predicted by the function $y_{D}^{(S u .)}\left(\theta_{i . D}^{(S u .)}\right)$ - experimental results of fig. 9 are predicted qualitatively by fig. $1 \mathrm{~B}$ and the corresponding equations.

The determination of the stationary growth rate $v_{g r}^{*}$ is the first step for the characterization of the LPE process. The stationary growth rate can be determined by the slope of the linear part of the curve $d(t)$ at great durations of the growth process $(t>\tau)$. By determining the intersection with the ordinate axis $d^{(S u .)^{* *}}$ and using equation 27 the time constant $\tau$ can be determined. Then, using equations 29-31, all the other parameters can be obtained.

In eq. 18 there is a second time constant $-\tau_{00 i . D}=1 / G_{i . D}=\Delta C_{i . D}^{L} \tau_{0 D}$. Each time constant is in relation to some process. The type of process thet characterizes this constant has not been determined at this time. An approximate assessment of its magnitude provides that this constant is 3-4 orders of magnitudes less than the time constant of the growth process itself $\tau_{0 D}^{(S u .)}$. It means that the constant $\tau_{00 i . D}$ refers to a very fast process that is much faster than the transition growth process, characterized by $\tau_{0 D}^{(S u .)}$. We presume the constant probably relates to the kinetics taking place on the crystal surface. Both constants 
are in proportional relation: $\tau_{0 D}=\left(k_{i} / v_{T} G_{i . D}\right)^{1 / 2}=\left(k_{i} \tau_{00 i . D} / v_{T}\right)^{1 / 2}$. Last relation shows that the product $k_{i} \tau_{00 i . D}$ is constant over all components:

$$
k_{i} \tau_{00 i . D}=v_{T} \tau_{0 D}^{2}=\tau_{0 D} \Delta T^{*}=\Theta_{0 D}=\text { const. }
$$

\section{Conclusions}

The presented approach for investigation of the liquid phase epitaxial growth process allows the determination of the most important parameters which characterize both the transition regime of growth and the stationary one at both considered growth mechanisms and for all three techniques. The theoretically established character of the dependence of the growth layer thickness on the duration of growth is confirmed by the experiments. Such confirmation has been shown for the time dependence of the averaged growth rate in case of supercooling technique for the dislocation mechanism. Good agreement between two experimental curves (the grown layer thickness versus time and the averaged growth rate versus time) and the theoretical ones is observed using the obtained values of the parameters and applying the equations for the dislocation growth mechanism. One may conclude that in our experiments the layer growth occurs by the dislocation mechanism because of the better reproduction of the experimental results. The presented approach may be applied for an exhaustive investigation of the LPE growth and allows better understanding of the process.

\section{References}

[1] T. Aichele, A. Lorenz, R. Hergt, P. Görnert, Cryst. Res. Technol. 38, No. 7-8, 575 587 (2003).

[2] B. Keszei, Z. Vertesy, G. Vertesy, Cryst. Res. Technol. 36, No. 8-10, 953-959 (2001).

[3] V. Gorbenko, Yu. Zorenko, V. Savchyn, T. Zorenko, A. Pedan, V. Shkliarskyi, Radiation Measurements 45, 461-464 (2010).

[4] Fumio Kawamura, Tomoya Iwahashi, Kunimichi Omae, Masanori Morishita, Masashi Yoshimura, Yusuke Mori and Takatomo Sasaki, Jpn. J. Appl. Phys. 42, pp. L4-L6 (2003).

[5] Fumio Kawamura, Hidekazu Umeda, Minoru Kawahara, Masashi Yoshimura, Yusuke Mori, Takatomo Sasaki, Hideaki Okado, Kazuto Arakawa and Hirotaro Mori, Jpn. J. Appl. Phys. 45, pp. 2528-2530 (2006).

[6] Fumio Kawamura, Tomoya Iwahashi, Masanori Morishita, Kunimichi Omae, Masashi Yoshimura, Yusuke Mori and Takatomo Sasaki, Jpn. J. Appl. Phys. 42, pp. L729-L731 (2003).

[7] Masato Aoki, Hisanori Yamane, Masahiko Shimada, Takashi Sekiguchi, Takashi Hanada, Takafumi Yao, Seiji Sarayama and Francis J. DiSalvo, Journal of Crystal Growth 218, 1, p. 7-12 (2000). 
[8] Fumio Kawamura, Hidekazu Umeda, Masanori Morishita, Minoru Kawahara, Masashi Yoshimura, Yusuke Mori, Takatomo Sasaki and Yasuo Kitaoka, Jpn. J. Appl. Phys. 45, pp. L1136-L1138 (2006).

[9] P. Benema, J. Crystal Growth 24/25, 1, 76 (1974).

[10] V. M. Andreev et. al., Liquid Phase Epitaxy in the Technology of Semiconductor Devices (Sovetskoe Radio, Moscow, 1975).

[11] S. Nakayama, M. Kaneko, S. Aizawa, K. Kashiwa, N. S. Takahashi, Journal of Crystal Growth 236, (1-3), pp 132-136 (2002).

[12] S. Dhar, Bull. Mater. Sci. 28, No. 4, pp. 349-353 (2005).

[13] S. Binetti, S. Pizzini, A. Cavallini, B. Fraboni, Physics and Techniques of Semiconductors 33, No. 6 (1999).

[14] G. H. B. Thompson, P.A. Kirkby, J. Crystal Growth 27, 70 - 80 (1974).

[15] J. I. Alferov et. al., Techn. Phys. Lett. 14, 1, 76 (1988).

[16] J. I. Alferov et. al., Technical Physics Letters 14, 2, 171 (1989).

[17] D. I. Wynne, C. S. Tull, E. E. Haller, MRS Proceedings 487, (1997).

[18] Xin Yao, Teruo Izumi, Yuh Shiohara, Physica C 386, 374-378 (2003).

[19] K. Kakimoto, Y. Sugawara, T. Izumi, Y. Shiohara, Physica C 334, pp. 249-258 (2000).

[20] Y. Shiohara in Proceedings of the 10th International Simposium on Superconductivity (ISS'97) "Advances in Superconductivity - X", Gifu, Oktober 27-30, 1997.

[21] T. Izumi, X. Yao, N. Hobara, K. Kakimoto, K. Haegawa,Y. Nakamura, T. Izumi, Y. Shiohara, IEEE Transactions on Applied Superconductivity 11, 1, p. 3481-3484. (2001).

[22] X. Yao, T. Izumi, Y. Shiohara, Physica C 386, pp. 374-378 (2003).

[23] T. Kawaguchi in "Fiber Crystal Growth from the Melt", Editor-in Chef T. Fukuda, P. Rudolph, S. Uda (Springer Verlag, Berlin, Heidelberg, New York, 2004).

[24] T. Kawaguchi, M. Imaeda, R. Ohuchi, M. Minakata, T. Taniuchi, T. Fukuda, J. Cryst. Gr. 166, 493 (1996).

[25] Y. Ishida, T. Kimura, K. Kakimoto, Y. Yamada, Z. Nakagawa, Y. Shiohara, A. B. Sawaoka, Physics C 292, p. 264-272 (1997).

[26] J. Figueras, T. Puig, X. Obradors, A. Erb, E. Walker, Phys. Rev. B 65, 092505 (2002).

[27] Y. Yamada and Y. Shiohara, Physica C 217, 182-188 (1993).

[28] P. Rogin, J. Hulliger, Journal of Crystal Growth 179, 551-558 (1997).

[29] T. Kawaguchi, D.-Ho Yoon, M. Minakata, Y. Okada, M. Imaeda, T. Fukuda, Journal of Crystal Growth 152, 87-93 (1995). 
[30] J. J. Hsieh, J. Cryst. Gr. 27, 49 (1974).

[31] D. Dutartre and M. Gavand, Revue, Phys. Appl. 19, 21-25 (1984).

[32] N. S. Peev, Crystal Research and Technology 45, No. 6, 593 - 599 (2010).

[33] N. S. Peev, Crystal Research and Technology 48, 2, p. 116-126 (2013).

[34] N. S. Peev, Crystal Research and Technology 25, 6, p. 653 (1990).

[35] N. S. Peev, Crystal Research and Technology 25, 11, pp. 1265-1269 (1990).

[36] N. S. Peev, Crystal Research and Technology 34, 7, pp. 851-858 (1999).

[37] N. S. Peev, Journal of Research in Physics 29, No. 2, p. 85-106 (2003).

[38] N. Peev, Journal of Research in Physic 34, No. 1, (2010).

[39] N. S. Peev, Journal of Crystal Growth 98, p. 499 (1989). 